\title{
A new saddle for the Dutch walking aid
}

\author{
A.W. Walther \\ Sirtemastraat 136, 2513 SR The Hague, The Netherlands \\ E-mail: walther-zillig@freeler.nl
}

In The Netherlands around 1960, Cahn designed a walking aid for people with walking difficulties. The idea was based on the old Gennan Draisine, a bike without pedals. The aid was developed and nowadays three models are available and can be supplied within the framework of the Dutch health laws.

The main problem of the present aid is the uncomfortable shape of the most saddles. The manufacturers of the aids use conventional cycle saddles, but unfortunately they are most uncomfortable for walking. Consequently, many new users of the aid are disappointed when they use the converted bicycle. They use it less and less and more often than not they give up. The only way to make the aid attractive to the increasing number of older people with walking difficulties would be to improve the comfort of the saddle. An attempt was made in 1999 to launch a research project with a view to developing a suitable saddle. To secure a grant from the EEC for this research a manufacturer had to be found with whom to co-operate, but no manufacturer was willing to do so because the demand for saddles would be far too limited.

Recently, a prototype of an improved saddle was developed for use with the Van Raam 1 walking aid. It has been tested extensively and was not painful, even after very long walks. The idea of the design is to separate the vertical load from the ischial bones from the horizontal load from the pubic bone by providing two separate sections, a sitting half and a forward half, thus removing the load from the pubic region.

The prototype is as illustrated in Fig. 1. The base of a bicycle saddle with three springs is fitted to the saddle-pin. A piece of plywood carrying the two parts of the saddle replaces the original saddle. In the tests it appeared that a fairly soft well-sprung sitting section is required. The prototype has a layer of $3-\mathrm{cm}$ thick
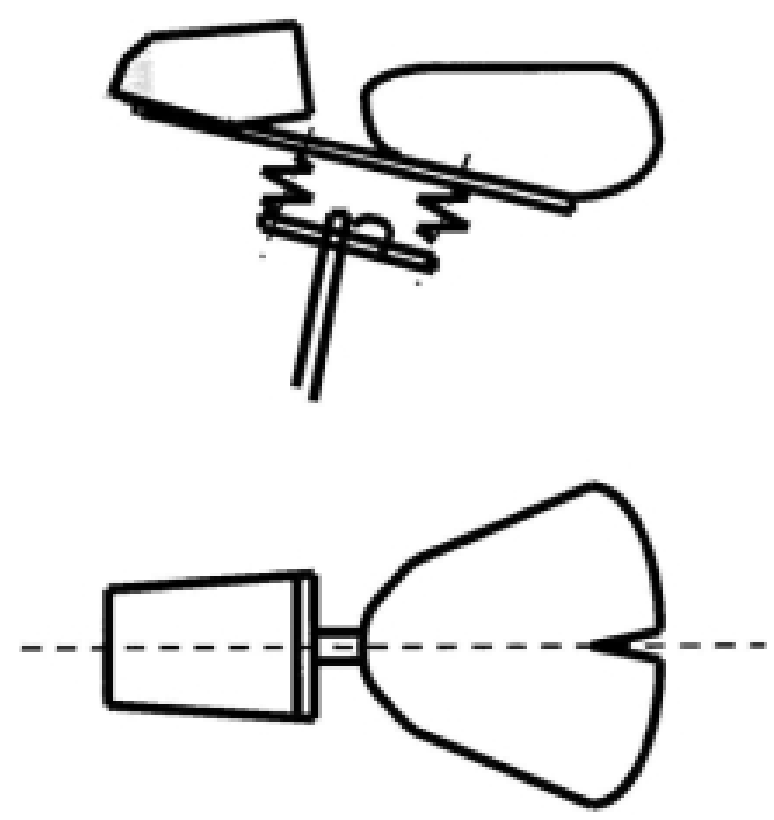

Fig. 1.

polystyrene sunnounted by a layer of 5-cm thick perforated foam latex. At the back of the sitting part a small recess prevents pain near the coccyx. The cover of this part of the saddle consists of $2-\mathrm{cm}$ thick latex and thin leather sheeting.

The front part of the prototype consists of an almost vertical wooden support covered with $2-\mathrm{cm}$ thick 'pressfoam' and 5-cm thick tempur. The front of this part of the saddle is covered with pressfoam and is slanted to facilitate mounting. The front is also covered with leather. The next step will be further development of the saddle and trying to find a manufacturer. It may be necessary to make the position of the front part adjustable in relation to the sitting part, because 
the dimensions of the pelvic bones vary. Meanwhile, every user of the walking aid must now have his own comfortable saddle made by a handy friend or by an upholsterer.

\section{Editor's comment}

As typical a Dutch invention as it seems: a bike for walking. But the walking bike is also used in other countries (see for example: www.walkwheel.de).
There is at least one major problem with these devices and that is the seat or saddle. When using saddle for a long period it is difficult enough to make it comfortable; it is even more difficult to get one which is designed for use on a walking bike, where control of forward movement and steering are additional functions of the saddle. In the technical note here, the author proposes a solution; readers are invited to comment on this solution.

MS 